\title{
Features of the interaction of the effector genes ToxA and ToxB with the susceptibility genes Tsn1 and Tsc2 in different species of wheat
}

\author{
Mironenko N. ${ }^{1 *}$, Baranova O. ${ }^{1}$, Kovalenko N. ${ }^{1}$, Mitrofanova O. ${ }^{2}$ \\ ${ }^{1}$ All-Russian Institute of Plant Protection, St. Petersburg, Russia \\ ${ }^{2}$ N.I. Vavilov All-Russian Institute of Plant Genetic Resources (VIR), St. Petersburg, Russia \\ *e-mail: nina2601mir@mail.ru
}

The Triticum aestivum-Pyrenophora tritici-repentis pathosystem is well studied in durum and common wheat. The purpose of our study is to assess the distribution of the sensitivity genes $T s n 1$ (5BL) and Tsc2 (2BL) in species of the genus Triticum L. from the VIR collection and the response of wheat accessions containing these genes to infection by isolates with complementary genes effectors ToxA and ToxB. All 72 accessions of 16 wheat species were evaluated for resistance to two isolates Tox $\mathrm{A}^{+}$originating from Kazakhstan and Russia, and one $\mathrm{ToxB}^{+}$from Greece. Using gene-specific primers, $T s n 1$ and $T s c 2$ were not detected in the diploid species T. urartu, T. boeoticum, and $T$. monococcum. In the wild tetraploid wheats $T$. dicoccoides and $T$. araraticum and six cultivated tetraploid species, the $T_{s c} 2$ gene and the polymorphism of the $T_{s n} 1$ were detected. In the T. timopheevii, only the $T s c 2$ gene was identified. Polymorphism for both genes was observed in all hexaploid species (genome $B B A A D D$ ). The manifestation of necrosis and/or chlorosis on wheat leaves is observed when the plant and the pathogen have both of the dominant genes $T s n / T o x A$ and/or $T s c 2 / T o x B$, respectively. All diploid wheat species had no susceptibility reactions. The gene-on-gene $T_{s c} 2 / \operatorname{ToxB}$ gene interaction was observed in accessions of T. aethiopicum and T. turgidum, as well as for most accessions of hexaploid species. All accessions of $T$. dicoccoides and $T$. dicoccum, despite the presence of the $T s c 2$ gene, were resistant to the $\mathrm{ToxB}^{+}$isolate. The reasons for this resistance are being studied. When evaluating the interaction of the $T s n 1 / \operatorname{ToxA}$, 11 accessions of different tetra- and hexaploid species of wheat aroused particular interest. The Tsn 1 was not detected in these accessions, but a strong necrosis was observed when infected with ToxA $\mathrm{A}^{+}$isolate, which is possibly due to the presence of other unknown susceptibility and effector genes.

Acknowledgements: This study was partially supported by the RFBR grant 18-04-00128a. 